\title{
Nucleon-nucleon bremsstrahlung: An example of the impossibility of measuring off-shell amplitudes
}

\author{
Harold W. Fearing \\ TRIUMF, 4004 Wesbrook Mall, Vancouver, B.C., Canada V6T $2 A 3$
}

(May 8, 2018)

\begin{abstract}
For nearly fifty years theoretical and experimental efforts in nucleon-nucleon bremsstrahlung $(\mathrm{NN} \gamma)$ have been devoted to measuring off-shell amplitudes and distinguishing among various NN potentials on the basis of their off-shell behavior. New experiments are underway, designed specifically to attain kinematics further off shell than in the past, and thus to be more sensitive to the off-shell behavior. This letter shows that, contrary to these expectations, and due to the invariance of the S-matrix under transformations of the fields, the off-shell NN amplitude is as a matter of principle an unmeasurable quantity in $\mathrm{NN} \gamma$.
\end{abstract}

13.75.Cs,25.20.-x,25.40.-h 
The nucleon-nucleon bremsstrahlung ( $\mathrm{NN} \gamma$ ) reaction, specifically $p+p \rightarrow p+p+\gamma$, was originally proposed [1] as a way of determining off-shell aspects of the nucleon-nucleon $(\mathrm{NN})$ force and distinguishing among different NN potentials. Since the original suggestion, there have been many calculations, Refs. [2 - 5], mostly in non-relativistic potential models. Recent experiments, e. g. [6], unlike earlier ones, seem to show that within the context of contemporary potential models, off-shell effects are important. However most modern potentials seem to have similar off-shell behavior in the region which has been explored, and thus it has not yet been possible to distinguish among potentials.

New experiments underway [7] will provide data in new kinematic regions and with much better accuracy than before. While there have been some new motivations to look at $\mathrm{NN} \gamma$, notably the fact that it may be a useful probe of heavy ion reactions, these new experiments have all been designed to explore kinematics further off shell than before so that they will be more sensitive to off-shell effects. Thus a primary aim has been to distinguish among potentials via their off-shell behavior, in accord with the established expectations for $\mathrm{NN} \gamma$.

The aim of this paper is to show that, in contrast to these expectations, in actual fact the off-shell NN amplitude is unmeasurable in $\mathrm{NN} \gamma$. This is true in principle and follows from the invariance of the S-matrix under transformations of the fields. This result has profound implications for it means that much previous work on $\mathrm{NN} \gamma$, aimed at determining the off-shell NN amplitude and distinguishing among potentials, was in fact misguided. The principle is general, and thus $\mathrm{NN} \gamma$ here serves as an example of the unmeasurability of off-shell effects.

To make a convincing case for this result it is necessary to understand in a qualitative way the ambiguities of the usual non-relativistic potential model approach. One must also understand why this result has not been noticed in the past. It turns out that inherent approximations in potential models camouflage the effect. Hence to proceed further, a simple field theory model is described in which everything can be calculated and in which one can see rigorously exactly how the off-shell NN amplitude enters and why it is not a measurable quantity.

In the context of a non relativistic potential model one can rigorously define and calculate, say by solving the Lippman-Schwinger equation, both an on- and off-shell NN amplitude. To connect this off-shell amplitude with $\mathrm{NN} \gamma$ experiments one uses it in the external radiation graph in which a photon is simply attached to the external legs, as in Fig. Ia and its permutations. Modern calculations also include the so-called double scattering contribution, Fig. [1 b which is typically a 10-30\% contribution. The full $\mathrm{NN} \gamma$ amplitude also involves a piece with off-shell effects in the electromagnetic interaction, as in Fig. 11c, which has been considered by only a few authors, [8].

Another contribution is a contact term, Fig. 11d, which reflects radiation from the charged lines interior to the strong interaction and is necessary to preserve gauge invariance. A simple example of such a contribution is given in Fig. 2. In a potential model it is impossible to calculate such contributions rigorously. Even the most microscopic potentials have a large number of phenomenological components, e. g. form factors, for which the underlying currents are not known. One can fix part of the leading contribution to this contact term by some variant of a soft photon approximation, and some explicit exchange contributions can be calculated [4, [5, 95]. However in most potential model calculations most contributions of this contact term are just neglected. 
Before proceeding to more rigorous analysis it is important to get a qualitative understanding of the reasons off-shell effects are unmeasurable. The off-shell NN amplitude can be written schematically as $T=T_{o n}+\left(p^{2}-m^{2}\right) T_{\text {off }}$ where $T_{o n}$ is the on-shell amplitude and $\left(p^{2}-m^{2}\right) T_{o f f}$ is the off-shell part which vanishes when the particle goes on shell, $p^{2}=m^{2}$. When this off-shell amplitude is put into the bremsstrahlung amplitude of Fig. [la however, the $\left(p^{2}-m^{2}\right)$ factor is exactly cancelled by the propagator. Thus the part involving $T_{o f f}$ looks just like a contact term and could have been included in the contact term. So there is an obvious ambiguity in that off-shell effects could be considered part of the external radiation diagram or part of the contact term.

A second important observation arises from the field theory result [10 that it is possible to make transformations on the fields in the theory, which will change the interaction, but will not change any physical quantity, in particular any S-matrix element.

The third observation is that it is the terms in the interaction proportional to the equation of motion (EOM) which are changed by such transformations [11]. In the present case the EOM generates (in momentum space and for spin zero particles) the off-shell factor $\left(p^{2}-m^{2}\right)$. Thus field transformations in effect can arbitrarily change the coefficient of the off-shell contribution without changing any of the physical results.

Now it is possible to understand in a qualitative sense the problem with the off-shell contributions. At the simplest level there will always be an ambiguity in these off-shell terms because they can also be written as contact terms. Usually such contact terms are dropped, so one gets different off-shell dependences depending on how much of the off-shell term is included in the terms kept.

At a more fundamental level one can always make a transformation of the fields which changes the coefficient of the EOM terms in the interaction and hence the coefficient of the off-shell NN contributions to the $\mathrm{NN} \gamma$ amplitude (Fig. 1a). Since this transformation does not change the $\mathrm{S}$-matrix it does not change the measurable $\mathrm{NN} \gamma$ amplitude. Thus a measured $\mathrm{NN} \gamma$ amplitude corresponds to any of an infinite selection of coefficients of the off-shell part of the amplitude. It thus follows that such off-shell amplitudes are simply not measurable.

Furthermore since the $\mathrm{NN} \gamma$ amplitude cannot depend on the transformation, it cannot depend at all on the coefficients of the EOM terms in the Lagrangian, since one possible transformation would make all such coefficients zero. Thus such terms must cancel exactly between off-shell parts appearing in the diagrams of Fig. 1 $\mathrm{a}$ and the contact term of Fig. I d.

There may also be off-shell contributions from parts of the interaction which do not come directly from the EOM terms in the Lagrangian. Such terms cannot be separated in the amplitude from the EOM terms and so cannot be separately measured. Furthermore they come from pieces of the interaction which are already determined by on-shell information only.

It is interesting to speculate on why this scenario has not been realized in the past. In part it must be because it depends crucially on an understanding of cancellations between the main terms and the contact term, which is essentially impossible to calculate in potential models and so is usually dropped at the very beginning. Furthermore, the concept of field transformations and invariance of the S-matrix under such transformations is really a field theory concept somewhat distant from the concepts familiar in non relativistic potential model calculations. There have been however a few similar observations for related processes. 
For example Scherer and Fearing [12,13] showed that off-shell effects did not contribute to Compton scattering on the pion and others [14] have discussed similar concepts with respect to the pion-nucleon system.

For completeness note that off-shell vertices are often simply parameterized, rather than calculated from the interaction. These parameters become a prescription accounting for unknown contact terms and other approximations in the model, and clearly can be obtained from measurable results. However they bear no direct relation to the 'physical' off-shell amplitudes calculated directly from the interaction which are being discussed here.

To make this qualitative understanding more rigorous, consider a model which allows a complete calculation of the contact terms and in which it is possible to look at the effects of field transformations explicitly. The standard potential model approach allows neither. The model is a simple field theory for spin zero particles, only one charged. With one minor exception to be discussed below, spin is really irrelevant. Thus the prototype reaction is $\pi^{+}+\pi^{0} \rightarrow \pi^{+}+\pi^{0}+\gamma$. Take as the Lagrangian for this reaction $\mathcal{L}=\mathcal{L}_{2}+\mathcal{L}_{4}^{G L}+\Delta \mathcal{L}_{4}$, where

$$
\mathcal{L}_{2}=\frac{F_{0}^{2}}{4} \operatorname{Tr}\left(D_{\mu} U\left(D^{\mu} U\right)^{\dagger}\right)+\frac{F_{0}^{2}}{4} \operatorname{Tr}\left(\chi U^{\dagger}+U \chi^{\dagger}\right)
$$

$D_{\mu}$ is a covariant derivative, $\chi$ involves the masses, $U$ contains the pion fields via $U=$ $\exp \left[i \tau \cdot \pi / F_{0}\right]$, and $\mathcal{L}_{4}^{G L}$ is the usual Gasser-Leutwyler Lagrangian [15]. $\Delta \mathcal{L}_{4}$ contains the two allowed EOM terms as described in Ref. [16], namely

$$
\Delta \mathcal{L}_{4}=\beta_{1} \operatorname{Tr}\left(\mathcal{O O}^{\dagger}\right)+\beta_{2} \operatorname{Tr}\left[\left(\chi U^{\dagger}-U \chi^{\dagger}\right) \mathcal{O}\right]
$$

Here the $\mathcal{L}_{2}$ EOM is $\mathcal{O}=0=\left(D_{\mu} D^{\mu} U\right) U^{\dagger}-U\left(D_{\mu} D^{\mu} U\right)^{\dagger}-\chi U^{\dagger}+U \chi^{\dagger}+\frac{1}{2} \operatorname{Tr}\left(\chi U^{\dagger}-U \chi^{\dagger}\right)$.

This is just the chiral perturbation theory Lagrangian in the meson sector through $O\left(p^{4}\right)$. It should be strongly emphasized however that the results here have nothing to do with chiral perturbation theory as such. $\mathcal{L}$ is just an effective Lagrangian which, through $O\left(p^{4}\right)$, is the most general one satisfying gauge invariance and other appropriate symmetries. It is renormalizable to this order, but depends on the parameters in $\mathcal{L}_{4}^{G L}$ and on $\beta_{1}$ and $\beta_{2}$.

Using this Lagrangian the fully renormalized, irreducible, amplitude for the elastic process $\pi^{+}\left(p_{1}\right)+\pi^{0}\left(p_{2}\right) \rightarrow \pi^{+}\left(p_{3}\right)+\pi^{0}\left(p_{4}\right)$, with the charged pions off shell is

$$
\begin{aligned}
\Gamma_{4 \pi} & =\frac{i}{F_{0}^{2}}\left[T_{0}\left(p_{1}, p_{3}\right)-\frac{1}{3}\left(\Lambda_{1}+\Lambda_{3}\right)\right]+\frac{8 i m_{\pi}^{2} \beta_{2}}{3 F_{0}^{4}}\left(\Lambda_{1}+\Lambda_{3}\right) \\
& +\frac{16 i \beta_{1}}{F_{0}^{4}}\left[T_{0}\left(p_{1}, p_{3}\right)\left(\Lambda_{1}+\Lambda_{3}\right)-\frac{1}{3}\left(\Lambda_{1}^{2}+\Lambda_{3}^{2}\right)\right]
\end{aligned}
$$

where $T_{0}\left(p_{1}, p_{3}\right) \equiv T_{0}\left(p_{1}, p_{2}, p_{3}, p_{4}\right)=\frac{1}{2}\left[\left(p_{1}-p_{3}\right)^{2}+\left(p_{2}-p_{4}\right)^{2}\right]-m_{\pi}^{2}$ with $\Lambda_{i}=p_{i}^{2}-m_{\pi}^{2}$. The tree level terms coming from $\mathcal{L}_{4}^{G L}$ and the one loop terms involving $\mathcal{L}_{2}$ twice will never be written explicitly. Such terms are finite but irrelevant to the argument.

This amplitude involves an off-shell component, proportional to $\beta_{1}$ and $\beta_{2}$, coming from the EOM terms. There are also a few off-shell contributions from the first term, both proportional to $\left(\Lambda_{1}+\Lambda_{3}\right)$ and originating in the momentum dependence buried in $T_{0}$. However these terms depend only on quantities available from the on-shell amplitude. 
Now consider the most general field transformation to $O\left(p^{2}\right)$ 13 , $U \rightarrow \exp (i S) U$ where for arbitrary real $\alpha_{1}, \alpha_{2}$

$$
S=\frac{4 i}{F_{0}^{2}}\left[\alpha_{1} \mathcal{O}-\alpha_{2}\left(\chi U^{\dagger}-U \chi^{\dagger}-\frac{1}{2} \operatorname{Tr}\left(\chi U^{\dagger}-U \chi^{\dagger}\right)\right)\right] .
$$

When applied to the fields $U$ in $\mathcal{L}_{2}$ it generates a correction to the Lagrangian of $\mathcal{O}\left(p^{4}\right)$ given by $\delta \mathcal{L}_{2}=\alpha_{1} \operatorname{Tr}\left(\mathcal{O} \mathcal{O}^{\dagger}\right)+\alpha_{2} \operatorname{Tr}\left(\left(\chi U^{\dagger}-U \chi^{\dagger}\right) \mathcal{O}\right)$, which has exactly the same form as $\Delta \mathcal{L}_{4}$. Thus field transformations can be used to change the coefficients $\beta_{1}$ and $\beta_{2}$ appearing in the original Lagrangian to zero or to anything one wishes. Since such transformations do not effect the physical results, the bremsstrahlung amplitude calculated with this Lagrangian, cannot depend on the values of $\beta_{1}$ and $\beta_{2}$, which according to Eq. (1) are coefficients of off-shell parts of the elastic amplitude.

To see this in more detail, use this Lagrangian to calculate the full $\mathrm{NN} \gamma$ amplitude. The fully renormalized, irreducible electromagnetic vertex for $\pi^{+}\left(p_{i}\right) \rightarrow \pi^{+}\left(p_{f}\right)+\gamma(k)$ is given by 16]

$$
\Gamma_{\pi \pi \gamma}=-i e \epsilon \cdot\left(p_{i}+p_{f}\right)\left[1+\frac{16 \beta_{1}}{F_{0}^{2}}\left(\Lambda_{i}+\Lambda_{f}\right)\right]
$$

where as before $\Lambda=p^{2}-m_{\pi}^{2}$. Note the off-shell part $\sim \beta_{1}$. There are also corrections to the renormalized pion propagator coming from $\Delta \mathcal{L}_{4}$, [12]

$$
\Delta_{R}^{-1}(p)=\left(p^{2}-m_{\pi}^{2}\right)\left[1+\frac{16 \beta_{1}}{F_{0}^{2}}\left(p^{2}-m_{\pi}^{2}\right)\right]+i \epsilon .
$$

The corrections to the renormalized propagator cancel exactly the off-shell part of the electromagnetic vertex. This is a general result for spin zero particles and follows from the Ward-Takahashi identity [17] which implies that at $k^{2}=0$ the the full renormalized propagator times the half off-shell electromagnetic vertex is equal to the free propagator times the on-shell electromagnetic vertex (c.f. Appendix A of Ref. [16]). Thus for spin zero bremsstrahlung one never needs to consider off-shell effects at the electromagnetic vertex. For spin one half particles there may be terms in the vertex involving magnetic moments which are gauge invariant by themselves and so are not constrained by the Ward-Takahashi identity [17]. These may give residual off-shell terms at the electromagnetic vertex which would be treated exactly as those at the strong vertex.

Thus the amplitude for radiation from external legs is

$$
\begin{aligned}
M_{1+3} & =\frac{i e}{F_{0}^{2}}\left[T_{0}\left(p_{1}, p_{3}\right)-k \cdot\left(p_{1}-p_{3}\right)\right]\left(\frac{\epsilon \cdot p_{3}}{k \cdot p_{3}}-\frac{\epsilon \cdot p_{1}}{k \cdot p_{1}}\right) \\
& -\frac{2 i e}{3 F_{0}^{2}} \epsilon \cdot\left(p_{3}+p_{1}\right) \\
& \times\left[1-\frac{48 \beta_{1}}{F_{0}^{2}}\left[T_{0}\left(p_{1}, p_{3}\right)-k \cdot\left(p_{1}-p_{3}\right)\right]-\frac{8 m_{\pi}^{2} \beta_{2}}{F_{0}^{2}}\right] \\
& -\frac{64 i e \beta_{1}}{3 F_{0}^{4}}\left(\epsilon \cdot p_{3} k \cdot p_{3}-\epsilon \cdot p_{1} k \cdot p_{1}\right)
\end{aligned}
$$


The first term comes from the on-shell part of the strong amplitude. The rest arises from the off-shell part of the strong vertex, which cancels the propagator denominator and thus looks like a contact term.

In this model the double scattering term, Fig. [1 b, would be a one-loop contribution $O\left(p^{6}\right)$. Thus $M_{1+3}$ is the exact analogue of what is calculated in a non relativistic potential model. It contains off-shell terms depending on the parameters of the interaction $\beta_{1}$ and $\beta_{2}$. The standard argument of the usual approach would be that by measuring the $\mathrm{NN} \gamma$ amplitude and comparing it with the calculated $M_{1+3}$ one could determine $\beta_{1}$ and $\beta_{2}$ and thus distinguish among different interactions.

This cannot be correct however since the field transformations, which leave the measurable bremsstrahlung amplitude unchanged, can change the coefficients $\beta_{1}$ and $\beta_{2}$ arbitrarily. Thus the off-shell component of $M_{1+3}$, which in the spin one half case might come also from the electromagnetic vertex, cannot be a measurable quantity.

The resolution of this problem depends on the renormalized contact term which can be calculated explicitly in this model, unlike in the standard potential model calculations. It is given by

$$
\begin{aligned}
\Gamma_{4 \pi \gamma} & =\frac{2 i e}{3 F_{0}^{2}} \epsilon \cdot\left(p_{1}+p_{3}\right) \\
& \times\left[1-\frac{48 \beta_{1}}{F_{0}^{2}}\left[T_{0}\left(p_{1}, p_{3}\right)-k \cdot\left(p_{1}-p_{3}\right)\right]-\frac{8 m_{\pi}^{2} \beta_{2}}{F_{0}^{2}}\right] \\
& +\frac{64 i e \beta_{1}}{3 F_{0}^{4}}\left(\epsilon \cdot p_{3} k \cdot p_{3}-\epsilon \cdot p_{1} k \cdot p_{1}\right) .
\end{aligned}
$$

The $\beta_{1}$ and $\beta_{2}$ terms here cancel those in $M_{1+3}$ of Eq. (2) and the full amplitude is in fact independent of $\beta_{1}$ and $\beta_{2}$ as it must be. In this case even the single off-shell term appearing in $\Gamma_{4 \pi}$ which is independent of $\beta_{1}$ and $\beta_{2}$ cancels, though that is probably not a general result. The final amplitude thus involves off-shell effects only via the momentum dependence implicit in $T_{0}$, which is completely determined by the on-shell amplitude.

Some further insight can be obtained from the soft photon limit of the model amplitude, since it reflects a model-independent imposition of gauge invariance, which enforces some of the cancellation between external radiation and contact terms. One sees from Eq. (2) that the $O(1 / k)$ part of $M_{1+3}$ is gauge invariant. The rest of the first term, which has all the $O(k / k)$ parts, is also gauge invariant. This implies that the remaining $O\left(k^{0}\right)$, independent of $k$ terms which are fixed by gauge invariance, must be zero. That then enforces the cancellation of most of the off-shell terms between this amplitude and the contact amplitude of Eq. (3) leaving just a few $O(k)$ terms not fixed by gauge invariance.

The results of this investigation can be summarized as follows. Field transformations change coefficients of off-shell parts of the elastic amplitude without changing the bremsstrahlung amplitude. Thus these off-shell amplitudes are unmeasurable in $\mathrm{NN} \gamma$, contrary to widespread expectations. Invariance under these transformations means also that there will be large cancellations between usual external radiation diagrams and the contact term, which is normally not calculated in standard potential approaches. Thus microscopic type calculations which allow a complete evaluation of the contact term should be favored. At the very least, in potential model calculations some technique which enforces gauge invariance and the cancellations that entails, at least at soft photon order, should be used. 
None of this means that $\mathrm{NN} \gamma$ is less interesting. Quite the contrary, since it is now clear that a full understanding of the process requires a detailed understanding of the contact terms, which involve a photon probe of the inner details of the NN interaction. Finally, none of these considerations are particularly specific to $\mathrm{NN} \gamma$. It is probable that off-shell amplitudes are unmeasurable in any process and calculations purporting to show sensitivity of physically measurable quantities to such off-shell effects should be viewed with suspicion.

The author would like to thank Stefan Scherer for a very careful reading of the manuscript, for some useful comments, and for what has now been some years of fruitful collaboration on these and related topics. 


\section{REFERENCES}

[1] J. Ashkin and R. Marshak, Phys. Rev. 46, 58 (1949); Erratum, ibid, 989.

[2] H. W. Fearing, Nucl. Phys A463, 95 (1987); R. L. Workman and H. W. Fearing, Phys. Rev. C 34, 780 (1986).

[3] See e.g. V. Brown, P. L. Anthony, and J. Franklin, Phys. Rev. C 44, 1296 (1991); V. Herrmann, K. Nakayama, O. Scholten, H. Arellano, Nucl. Phys. A582, 568 (1995); A. Katsogiannis and K. Amos, Phys. Rev. C 47, 1376 (1993); M. Jetter and H. V. von Geramb, Phys. Rev. C 49, 1832 (1994); G. H. Martinus, O. Scholten, and J. A. Tjon, Phys. Lett. B 402, 7 (1997).

[4] M. Jetter and H. W. Fearing, Phys. Rev. C 51, 1666 (1995).

[5] J. A. Eden and M. F. Gari, Phys. Rev. C 53, 1102 (1996).

[6] K. Michaelian et al., Phys. Rev. D 41, 2689 (1990); P. Kitching, et al., Phys. Rev. Lett. 57, 2363 (1986); Nucl. Phys. A463, 87 (1987).

[7] E. Kuhlmann, N. Kalantar, J. Matthews, J. Zlomanczuk, M. Nomachi, private communications.

[8] E. M. Nyman, Nucl. Phys. A154, 97 (1970); A160, 517 (1971); S. Kondratyk, G. Martinus, O. Scholten, Phys. Lett. B 418, 20 (1998).

[9] F. de Jong and K. Nakayama, Phys. Rev. C 52, 2377 (1995).

[10] S. Kamefuchi, L. O'Raifeartaigh and Abdus Salam, Nucl. Phys. 28, 529 (1961); J. Chisholm, Nucl. Phys. 26, 469 (1961); R. Haag, Phys. Rev. 112, 669 (1958).

[11] S. Weinberg Quantum Theory of Fields, vol. 1, p. 331 (Cambridge Univ. Press, 1995).

[12] S. Scherer and H. W. Fearing, Phys. Rev. C 51, 359 (1995); S. Scherer, Proc. of the Conf. on Perspectives in Nuclear Physics at Intermediate Energies, Trieste, May, 1995, nucl-th/9506028.

[13] S. Scherer and H. W. Fearing, Phys. Rev. D 52, 6445 (1995).

[14] R. M. Davidson and G. I. Poulis, Phys. Rev. D 54, 2228 (1996); J. Adam, F. Gross, J. W. Van Orden, nucl-th/9708002.

[15] J. Gasser and H. Leutwyler, Ann. Phys. 158, 142 (1984).

[16] T. E. Rudy, H. W. Fearing, and S. Scherer, Phys. Rev. C50, 447 (1994).

[17] J. C. Ward, Phys. Rev. 78, 1824 (1950); Y. Takahashi, Nuovo Cimento 6, 370 (1957). 


\section{FIGURES}

FIG. 1. (a) External radiation graph off shell at the strong vertex; (b) The double scattering contribution; (c) A contribution off shell at the electromagnetic vertex; (d) The contact term.

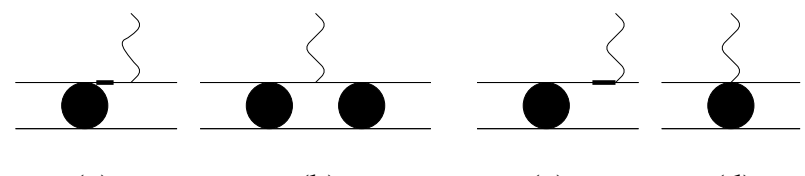

(a)

(b)

(c)

(d)

FIG. 2. A typical contribution to the strong potential (a), leading to a usually neglected contribution (b) to the contact term of the $\mathrm{NN} \gamma$ amplitude.

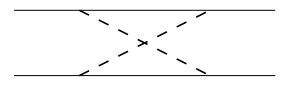

(a)

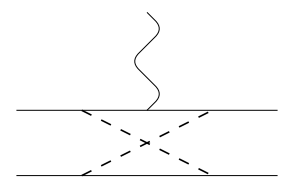

(b) 